\title{
Structural Characterization of the Helicobacter pylori Peptidyl-Prolyl cis,trans- Isomerase HP0175 Reveals an Extension of Chaperone Helices Upon Target Interaction
}

\author{
Ayat Yaseen \& Gerald F. Audette \\ Dept. of Chemistry \& Centre for Research on Biomolecular Interactions \\ York University, 4700 Keele St., Toronto, ON, M3J 1P3
}

\begin{abstract}
Within a host, pathogenic bacteria employ several mechanisms that enhance their survival and motility. Helicobacter pylori secretes several virulence factors, including VacA, CagA, $\gamma$-glutamyltrasnpeptidase and HP0175. The secreted HP0175 has been shown to induce cell apoptosis through a cascade of mechanisms initiated through binding to Toll Like Receptor 4 (TLR4). HP0175 is classified as a parvulin-like peptidylprolyl cis,trans-isomerase (PPIase) involved in the isomerization of proline-containing peptide bonds. We have crystallized apo-HP0175 and determined the structure to $2.09 \AA$ resolution. A comparison to the indole-2-carboxylic acid bound HP0175 form of the protein reveals that the $\mathrm{N}$ - and $\mathrm{C}$-terminal helices of the chaperone domain extend upon engagement of the catalytic residues in the binding pocket by the inhibitor. Further, chromatographic and mass spectrometric analysis of HP0175 reveals that dimerization of the active form of the protein is dependent on ionic strength. These structural observations support the finding in other parvulins that the $\mathrm{N}$ - and C-terminal helices stabilize proteins undergoing catalysis by the PPIase domain, as well as suggest a means by which HP0175 could bind to TLR4, thereby initiating an apoptotic cascade in infected cells.
\end{abstract}

